\title{
Efficient assembly of full-length infectious clone of Brazilian IBDV isolate by homologous recombination in yeast
}

\author{
J.V.J. Silva Jr. ${ }^{1}$, S. Arenhart ${ }^{1,2}$, H.F. Santos ${ }^{3}$, S.R. Almeida-Queiroz ${ }^{1}$, A.N.M.R. Silva ${ }^{1}$, \\ I.M. Trevisol ${ }^{4}$, G.R. Bertani ${ }^{5}$, L.H.V.G. Gil ${ }^{1}$ \\ ${ }^{1}$ Departamento de Virologia e Terapia Experimental, Centro de Pesquisas Aggeu Magalhães, \\ Fundação Oswaldo Cruz, Recife, PE, Brazil. \\ ${ }^{2}$ Setor de Virologia, Departamento de Medicina Veterinária Preventiva, Centro de Ciências Rurais, \\ Universidade Federal de Santa Maria, Santa Maria, RS, Brazil. \\ ${ }^{3}$ Laboratório de Virologia, Departamento de Microbiologia, Imunologia e Parasitologia, \\ Instituto de Ciências Básicas da Saúde, Universidade Federal do Rio Grande do Sul, \\ Porto Alegre, RS, Brazil. \\ ${ }^{4}$ Embrapa Suínos e Aves, Concórdia, SC, Brazil. \\ ${ }^{5}$ Departamento de Bioquímica e Laboratório de Imunopatologia Keiso Asami, \\ Universidade Federal de Pernambuco, Recife, PE, Brazil.
}

Submitted: October 22, 2013; Approved: April 17, 2014

\begin{abstract}
The Infectious Bursal Disease Virus (IBDV) causes immunosuppression in young chickens. Advances in molecular virology and vaccines for IBDV have been achieved by viral reverse genetics (VRG). VRG for IBDV has undergone changes over time, however all strategies used to generate particles of IBDV involves multiple rounds of amplification and need of in vitro ligation and restriction sites. The aim of this research was to build the world's first VRG for IBDV by yeast-based homologous recombination; a more efficient, robust and simple process than cloning by in vitro ligation. The wild type IBDV (Wt-IBDV-Br) was isolated in Brazil and had its genome cloned in pJG-CMV-HDR vector by yeast-based homologous recombination. The clones were transfected into chicken embryo fibroblasts and the recovered virus (IC-IBDV-Br) showed genetic stability and similar phenotype to Wt-IBDV-Br, which were observed by nucleotide sequence, focus size/morphology and replication kinetics, respectively. Thus, IBDV reverse genetics by yeast-based homologous recombination provides tools to IBDV understanding and vaccines/viral vectors development.
\end{abstract}

Key words: infectious bursal disease virus, reverse genetics, yeast-based homologous recombination.

\section{Introduction}

Infectious bursal disease virus (IBDV) is a pathogen causing immunosuppressive disease in young chickens that multiplies rapidly in $\mathrm{B}$ lymphocytes precursors in the bursa of Fabricius (BF) (Jackwood et al., 1982). The IBDV infection may lead to immunosuppression and increased susceptibility to other diseases, causing significant losses to the poultry industry (Balamurugan and Kataria, 2006). In Brazil, the disease was first described by Nakano et al. (1972) through the pathological diagnosis. In 1978 Saukas and collaborators reported the virus isolation (Saukas, 1978) and cases of mortality due to very virulent IBDV (vvIBDV) were observed in this country in 1997 (Di Fabio et al., 1999).

IBDV is a member of the family Birnaviridae, genus Avibirnavirus and contains a double-stranded RNA (dsRNA) genome consisting of two segments designated A and B (Müller et al., 1979). The larger segment, A (3.2 kb), contains two partially overlapping open reading frames 
(ORFs 1 and 2). ORF1 encodes a 17-kDa nonstructural protein (VP5) present in IBDV infected cells (Mundt et al., 1995). ORF2 encodes for a precursor polyprotein (110-kDa), which is processed to produce the mature structural proteins VP2, VP3 and VP4 (Azad et al., 1985; Hudson et al., 1986). The smaller segment, B (2.8 kb), encodes VP1, a $97-\mathrm{kDa}$ multifunctional protein with polymerase and capping enzymatic activities (Pan et al., 2007; von Einem et al., 2004).

Reverse genetics has significantly increased the knowledge on several aspects relating to the IBDV. The first reverse genetics system to IBDV was created in 1996 with the generation of IBDV by cellular transfection of RNAs produced by in vitro transcription (Mundt and Vakharia, 1996). Advances came by the use of cloned viral cDNA transfection (Boot et al., 1999; Lim et al., 1999), which has been shown to be a better method than RNA transfection to rescue recombinant IBDV (Boot et al., 2001). The clone viral cDNA transfection method has passed for additional modifications (Ben Abdeljelil et al., 2008; Qi et al., 2007) and now has been used to study biological functions and vaccines development (Gao et al., 2011). Nowadays, the reverse genetics technology is the main tool for construction of IBDV recombinant attenuated vaccines, such as vector-based (Zanetti et al., 2012), chimeric vaccines (Gao et al., 2011) and virus-like particles (VLPs) production (Wang et al., 2012).

However, these different reported strategies of reverse genetics for IBDV involve multiple rounds of amplification, use of specific restriction sites and the need of in vitro ligation for assembly plasmids to generate IBDV particles. These strategies are tiresome, time consuming to perform and require many steps. However, in 1997, the observation that linear DNA fragments can stimulate recombination in Saccharomyces cerevisiae, led to a rapid development of methods for DNA manipulation in yeast (Oldenburg et al., 1997), such as yeast-based homologous recombination cloning technique. In this methodology, a DNA fragment containing homologous ends with the vector can be directly cloned using in vivo recombination in a linearized vector by an efficient, robust and simple process (Gibson, 2009; Shanks et al., 2009).

In the present study, we isolated IBDV from Brazilian commercial chicken (wild type IBDV-Br, Wt-IBDV-Br) with clinical signals suggestive of IBDV infection, amplified its genome and confirmed its identity by sequencing. After complete virus genome amplification, we built and characterized, in cell culture, the first reverse genetics system of IBDV made by homologous recombination in yeast. This new biotechnology tool can be used to study the IBDV molecular virology and new vaccines development.

\section{Material and Methods}

\section{Cell culture and IBDV Isolation}

Chicken embryo fibroblasts (CEF) cells (Embrapa Swine and Poultry, Santa Catarina, Brazil) were grown in F10/M199 medium containing 10\% fetal bovine serum (FBS) supplemented with 1\% Penicillin and Streptomycin (LGC Biotecnologia, Cotia, SP, Brazil), 0.025\% Neomycin Sulfate (LGC Biotecnologia, Cotia, SP, Brazil) and 0.1\% Fungizone (Gibco, Langley, OK, EUA). Cells were grown at $37^{\circ} \mathrm{C}$ in a humidified $5 \% \mathrm{CO}_{2}$ incubator (conditions maintained in all work).

The Wt-IBDV-Br strain was isolated from commercial chickens with signs of weight loss, BF irregular size, apathy and poor appetite. Spleen and BF from five of these commercial chickens with 26-27 days old with clinical signals suggestive of IBDV infection were processed in duplicate (Romero, 1987; Reynolds, 1998). The spleen and BF were macerated and suspended in sterile Phosphate Buffered Saline (PBS) 1/10 (weight/volume) supplemented with antibiotics (Romero, 1987; Reynolds, 1998). The macerated tissue was centrifuged at $3000 \mathrm{~g}$ for $15 \mathrm{~min}$ and $0.2 \mathrm{~mL}$ of supernatant was inoculated (in triplicate) in nine day old embryonated chicken eggs (Embrapa Swine and Poultry, Specific Pathogenic Free, SPF). The inoculation was performed in the chorioallantoic membrane (CAM), 0.2 $\mathrm{mL} /$ egg. After inoculation, eggs were incubated at $37^{\circ} \mathrm{C}$ and monitored for seven days by ovoscopy prior isolation steps. The negative control was performed with PBS inoculated in CAM of SPF eggs (Romero, 1987; Reynolds, 1998). The following steps for Wt-IBDV-Br isolation involved three successive inoculations in embryonated SPF eggs $(0.2$ $\mathrm{mL} / \mathrm{egg}$ ) using the chorioallantoic liquid as a virus source and then seven successive passages in CEF ( $1 \mathrm{~mL} /$ passage in $\mathrm{CEF}$ ) (titers were not measured between passages). The virus recovered in the last cell culture step was identified by sequencing of VP2 region and used to construct the reverse genetics system of IBDV.

The sequencing was performed with viral RNA (vRNA) extracted from $150 \mu \mathrm{L}$ of CEF cell culture supernatant infected with Wt-IBDV-Br by Viral RNA Isolation Kit (Macherey-Nagel, Düren, Germany) according to the manufacturer's protocol. vRNA was eluted from the column in a final volume of $50 \mu \mathrm{L}(160 \mathrm{ng} / \mu \mathrm{L})$ of RNase-free water and stored at $-80{ }^{\circ} \mathrm{C}$ until required.

The reverse transcription (RT) to VP2 region was performed in total $20 \mu \mathrm{L}$ reaction by mixing $2 \mu \mathrm{g}$ of vRNA with $5 \mu \mathrm{M}$ of VP2R primer (Table 1) and $200 \mathrm{U}$ of Superscript III RT (Invitrogen, Carlsbad, CA, USA) according to the manufacturer's protocol. The cDNA was amplified with Phusion High-Fidelity DNA Polymerase $100 \mathrm{U}(2 \mathrm{U} / \mu \mathrm{L}$ ) (Thermo Scientific, Waltham, MA, USA) according to the manufacturer's protocol. PCR's conditions were: $98^{\circ} \mathrm{C}$ for $30 \mathrm{~s}$, followed by 32 cycles of $98^{\circ} \mathrm{C}$ for $10 \mathrm{~s}, 54^{\circ} \mathrm{C}$ for $30 \mathrm{~s}, 72^{\circ} \mathrm{C}$ for 
Table 1 - Oligonucleotides used in amplification and cloning of IBDV segments into pJG-CMV-HDR vector ${ }^{\mathrm{a}}$.

\begin{tabular}{ll}
\hline Oligonucleotides & \multicolumn{1}{c}{ Sequence } \\
\hline IBDV A-F $^{\mathrm{b}}$ & TGGGAGGTCTATATAAGCAGAGCTCGTTTAGTGAACCGGGATACGATCGGTCTGACCC \\
IBDV A-R $^{\mathrm{c}}$ & GGAGGTGGAGATGCCATGCCGACCCGGGGACCCGCGAACGGATCC \\
IBDV B-F $^{\mathrm{b}}$ & TGGGAGGTCTATATAAGCAGAGCTCGTTTAGTGAACCGGGATACGATGGGTCTGACCC \\
IBDV B- $^{\mathrm{c}}$ & GGAGGTGGAGATGCCATGCCGACCCGGGGGCCCCCGCAGGCGAAG \\
VP2F $^{\mathrm{b}}$ & ACCATAAACGCCGTGACC \\
VP2R $^{\mathrm{c}}$ & CCGTGGATCGTCACTGCTA \\
\hline
\end{tabular}

${ }^{\mathrm{a}}$ Vector used for homologous recombination in yeast. The pJG-CMV-HDR vector has the cloning site between the transcription start site of the cytomegalovirus (CMV) promoter and the hepatitis delta virus (HDV) ribozyme sequence.

${ }^{\mathrm{b}}$ Forward primer.

${ }^{\mathrm{c}}$ Reverse primer.

${ }^{\mathrm{d}}$ Underlined sequences corresponding to the region used for homologous recombination in yeast.

$1 \mathrm{~min}$ and a final extension of $72{ }^{\circ} \mathrm{C}$ for $10 \mathrm{~min}$. The primers used to amplification step (VP2-F and VP2-R) are showed in Table 1.

After analyze of nucleotide sequence (made as described below), the identity of the isolated was confirmed by comparison between the obtained sequence and the available GenBank sequences (National Center for Biotechnology Information, $\mathrm{NCBI}$ ).

\section{Sequence analysis}

To confirm the sequence of Wt-IBDV-Br and validate plasmids constructs, nucleotide sequence was determined with the $\mathrm{ABI}$ BigDye terminator cycle sequencing on an $\mathrm{ABI}$ 3100 Genetic Analyzer (Applied Biosystems, Foster city, CA, USA). The sequence identity was confirmed by comparison amplified regions vs. NCBI sequences.

\section{Amplification of the segment $A$ and $B$}

After vRNA extraction from cell supernatant (160 ng/ $\mu \mathrm{L}), \mathrm{RT}$ to A segment was performed in total $20 \mu \mathrm{L}$ reaction with $5 \mu \mathrm{M}$ of IBDV A-R recombination reverse primer (Table 1). The RT was performed as describe in Material and Methods.

The cDNA for segment A was amplified in two overlapping PCR fragments (A1 and A2, overlapping by $639 \mathrm{nt}$ ) with Phusion High-Fidelity DNA Polymerase $100 \mathrm{U}$ (2 U/ $\mu \mathrm{L})$ (Thermo Scientific, Waltham, MA, USA) according to the manufacturer's protocol. PCR's conditions were: $98^{\circ} \mathrm{C}$ for $30 \mathrm{~s}$, followed by 32 cycles of $98^{\circ} \mathrm{C}$ for $10 \mathrm{~s}, 54^{\circ} \mathrm{C}$ for $30 \mathrm{~s}, 72^{\circ} \mathrm{C}$ for $3 \mathrm{~min}$ and a final extension of $72^{\circ} \mathrm{C}$ for 10 $\min$. The recombination primers used to amplify the A1 and A2 (IBDV A-F and VP2R; VP2F and IBDV A-R, respectively) and B (IBDV B-F and IBDV B-R) sequences are described in Table 1. The cDNA for segment $B$ was amplified in one fragment using the IBDV B-F and IBDV B-R primers and the same RT and PCR conditions described for segment A.

\section{Plasmid constructs}

The construction of the plasmid pJG-CMV-HDR has previously made by Gil and Silva (unpublished data), and was made by insertion of cytomegalovirus (CMV) promoter, a polylinker region and the hepatitis delta virus (HDV) ribozyme sequence into pB42AD vector (OriGene, Rockville, MD, USA) (data not shown). The pJG-CMVHDR plasmid has coding sequence for tryptophan (trp) amino acid as selectable marker gene for transformed cells selection on medium without trp. For cloning of IBDV infectious clone, the vector pJG-CMV-HDR was linearized with XhoI nuclease (Promega, USA) according to the manufacturer's protocol. The PCR products (A1, A2 and B) containing homologous ends sequences with linearized vector could be directly cloned downstream of the CMV promoter of the vector pJG-CMV-HDR to produce the recombinant plasmids pJG-IBDV-Br-A and pJG-IBDV-Br-B, respectively. All clones were generated by homologous recombination in Saccharomyces cerevisiae strain RFY206 (MATa, trp1, ura3-52, his3-200, leu2-3, lys2- $\Delta 201$, trp1::hisG). The selections of positive colonies (recombinated plasmid) were performed using Yeast Nitrogen Base (YNB) without trp (YNB - trp). The transformation of competent RFY206 was made by lithium acetate procedure as described previously (Sambrook and Russell, 2001). Positive colonies were grown YNB-trp and plasmid DNA extraction was performed using QIAprep Miniprep Kit according to manufacturer's instruction (Qiagen, Valencia, CA, USA). Colonies were screened by PCR to confirm the cloning. The PCR was performed with recombinant primers described in Table 1 (IBDV A-F/ IBDV A-R and IBDV B-F/ IBDV B-R to pJG-IBDV-Br-A and pJG-IBDV-Br-B, respectively). The PCR conditions were the same described in Material and Methods.

\section{Escherichia coli electroporation}

In order to amplify the DNA after homologous recombination, the purified yeast DNA was used to transform competent Escherichia coli strain DH10B (Invitrogen, Carlsbad, 
CA, USA). pJG-IBDV-Br-A and pJG-IBDV-Br-B DNA plasmids were electroporated using $2 \mathrm{~mm}$ cuvettes on an ECM BTX electro cell manipulator $830^{\mathrm{TM}}$ (BTX Instrument Division, Holliston, MA, USA) with the following settings: $2.75 \mathrm{kV}, 99 \mu \mathrm{s}$ and 5 pulses with $1 \mathrm{~s}$ interval. Individual colonies were grown at $37{ }^{\circ} \mathrm{C}$ overnight in LB medium with ampicillin. Plasmid DNA was then purified using a QIAGEN Plasmid Midi Kit (Qiagen, Valencia, CA, USA) according manufacturer's instructions.

\section{Transfection of CEF cells}

CEF cells were seeded at $7 \times 10^{5}$ cells $/$ well of a 6 -well tissue culture plate and cultured overnight. Cells were cotransfected with pJG-IBDV-Br-A and pJG-IBDV-Br-B using the PolyFect Transfection Reagent according to the manufacturer's protocol (Qiagen, Valencia, CA, USA) with $15 \mu \mathrm{L}$ of the reagent and $2.5 \mu \mathrm{g}$ of each DNA plasmid, measured by NanoPhotometer (Implen, Munich, Germany) according manufacturer's instructions. The negative control was performed with only the transfection of the pJG-IBDV-Br-A.

\section{Determination of IBDV cDNA infectious clone (IC-IBDV-Br) infectivity}

At 5 days post-transfection, the cell supernatant was collected (Passage 0; P0), and used to re-infect freshly prepared CEF cells for five additional serial passages (P1-P5). Verification of generated and infectious IC-IBDV-Br was made by cytopathic effect (CPE) in CEF cells and amplification of the A segment using extracted vRNA from cell supernatant P5. To avoid amplification of residual plasmid DNA used in the transfection, we included a negative control reaction without the reverse transcriptase.

\section{Phenotypic Characterization}

\section{Plaque Assay}

CEF cells were seeded in 6-well tissue culture plates (7 x $10^{5}$ cells/well) and incubated overnight. Monolayers were infected with serial dilutions of the parental virus (Wt-IBDV-Br) or IC-IBDV-Br $\left(10^{-1}\right.$ to $\left.10^{-9}\right)$. After $1 \mathrm{~h}$ absorption, the cells were washed once with PBS and overlaid with F10/199 medium containing 1\% agarose and 5\% FBS. After 3 days, cells were $10 \%$ formalin-fixed and revealed by $0.05 \%$ crystal violet.

\section{Growth curve of IBDV}

The replication kinetics of the IC-IBDV-Br vs. Wt-IBDV-Br was analyzed in CEF cells cultured in a 24well plate $\left(1.5 \times 10^{5}\right.$ cells/well). Cells were infected with Wt-IBDV-Br or IC-IBDV-Br virus at multiplicity of infection (MOI) of 0.03 . At time intervals of $12 \mathrm{~h}$ post-infection, the supernatants were collected and frozen at $-80^{\circ} \mathrm{C}$. The experiment was conducted for $72 \mathrm{~h}$. The infectious virus was titrated using plaque assay as described above. The result was obtained as mean of the two independent experiments. The titer was calculated based on a previously method (Reed and Muench, 1938).

\section{Genetic stability of the recovered virus}

To determine the genetic stability of the IC-IBDV-Br, its genome was compared to $\mathrm{Wt}-\mathrm{IBDV}-\mathrm{Br}$ clone by genomic alignment (Ape-A plasmid Editor v1.10.4).

\section{Results}

\section{Isolation and identification of the Wt-IBDV-Br}

The analysis of chicken embryo survival after virus inoculation is presented in Table 2. The dead embryos on the fourth day had a lower body development compared to control embryos, beyond bleeding in the skin, muscle and internal organs. All embryos had at least one of these lesions (data not shown). The CPE observed during all passages in CEF indicates that the Wt-IBDV-Br replicate efficiently in cell culture. The comparison Wt-IBDV-Br VP2 region $v s$. NCBI sequences confirmed the virus identity and identified the sequences in access number AJ310185.1 (access date $08 / 15 / 2012$ ) as the sequences of greatest homology (E value 0.0 and Identity $99 \%$ ).

\section{Construction of the full-length cDNA clone of IBDV genome}

To build the reverse genetics system, we have amplified and cloned the segment A and B. The segment A was amplified in two overlapping PCR fragments (A1 and A2) and segment $\mathrm{B}$ in one PCR product (Figure 1A). The amplified A1 (1173 bp), A2 (2789 bp) and B (2827 bp) were then assembled by homologous recombination into the pJG-CMV-HDR to produce $\mathrm{pJG}-\mathrm{IBDV}-\mathrm{Br}-\mathrm{A}$ and $\mathrm{pJG}-\mathrm{IBDV}-\mathrm{Br}-\mathrm{B}$ plasmids, respectively. The pJG-IBDV-Br-A and pJG-IBDV-Br-B plasmids were confirmed by full-length PCR (Figure 1B).

The comparison pJG-IBDV-Br-A and pJG-IBDV-Br-B plasmids $v s$. NCBI sequences identified the AJ310185.1 and EU162095.1 sequences (access date 08/15/2012) as the most homology to pJG-IBDV-Br-A (E value 0.0 and Identity $99 \%$ ) and pJG-IBDV-Br-B (E value 0.0 and Identity $99 \%$ ) respectively. Analysis of pJG-IBDV-Br-A and pJG-IBDV-Br-B vs. NCBI sequences are shown in Table 3.

\section{Identification of generated IBDV virus from plasmid constructs}

The IC-IBDV-Br identification was first performed by visualization of the $\mathrm{CPE}$ in cell culture. An additional verification was conducted by the segment A amplification (3260 bp) by RT-PCR of IC-IBDV-Br infected cells (Figure 2).

\section{Phenotypic characterization in cell culture}

The plaque assay confirmed the efficient replication of rescued virus in cell culture and shown morphology very similar to the Wt-IBDV-Br (Figure 3). The IC-IBDV-Br also exhibited growth kinetics similar to Wt-IBDV-Br (Figure 4). 
Table 2 - Analysis of embryo after inoculation with samples collected from chickens with suspected IBDV infection.

\begin{tabular}{|c|c|c|c|c|c|c|c|c|}
\hline \multirow[t]{2}{*}{ Chicken } & \multirow[t]{2}{*}{ Sample $^{\mathrm{a}}$} & \multicolumn{7}{|c|}{ Days post-inoculation $^{\mathrm{b}}$} \\
\hline & & $1^{\circ}$ & $2^{\circ}$ & $3^{\circ}$ & $4^{\circ}$ & $5^{\circ}$ & $6^{\circ}$ & $7^{\circ}$ \\
\hline \multirow[t]{4}{*}{1} & $\mathrm{BF}^{\mathrm{c}}-1$ & $-{ }^{e}$ & - & - & - & - & - & - \\
\hline & BF-2 & - & - & - & - & - & - & - \\
\hline & Spleen- $1^{\mathrm{d}}$ & $++^{\mathrm{e}}$ & & & & & & \\
\hline & Spleen-2 & - & - & - & + & & & \\
\hline \multirow[t]{4}{*}{2} & BF-1 & - & - & - & - & - & - & - \\
\hline & BF-2 & + & & & & & & \\
\hline & Spleen-1 & + & & & & & & \\
\hline & Spleen-2 & - & - & - & - & - & - & - \\
\hline \multirow[t]{4}{*}{3} & BF-1 & - & - & - & - & - & - & - \\
\hline & $\mathrm{BF}-2$ & - & - & - & - & - & - & + \\
\hline & Spleen-1 & + & & & & & & \\
\hline & Spleen-2 & - & - & - & - & - & - & - \\
\hline \multirow[t]{4}{*}{4} & BF-1 & + & & & & & & \\
\hline & $\mathrm{BF}-2$ & - & - & - & - & + & & \\
\hline & Spleen-1 & - & - & - & - & - & - & - \\
\hline & Spleen-2 & - & - & - & - & - & - & - \\
\hline \multirow[t]{4}{*}{5} & BF-1 & - & - & - & - & + & & \\
\hline & BF-2 & + & & & & & & \\
\hline & Spleen-1 & + & & & & & & \\
\hline & Spleen-2 & - & - & - & - & - & + & \\
\hline
\end{tabular}

${ }^{\text {a }}$ Samples collected from five commercial chickens with 26-27 days old.

${ }^{\mathrm{b}}$ Samples were inoculated in chorioallantoic membrane of Specific Pathogenic Free eggs with nine days old. After inoculation, eggs were monitored during seven days by ovoscopy looking for embryo moratlity.

${ }^{\mathrm{c}} \mathrm{BF}$ : bursa of Fabricius processed in duplicate (BF-1, BF-2).

${ }^{\mathrm{d}}$ Spleen processed in duplicate (Spleen-1, Spleen-2).

$\mathrm{e}_{+}$: dead embryos, -: embryo survival.

A

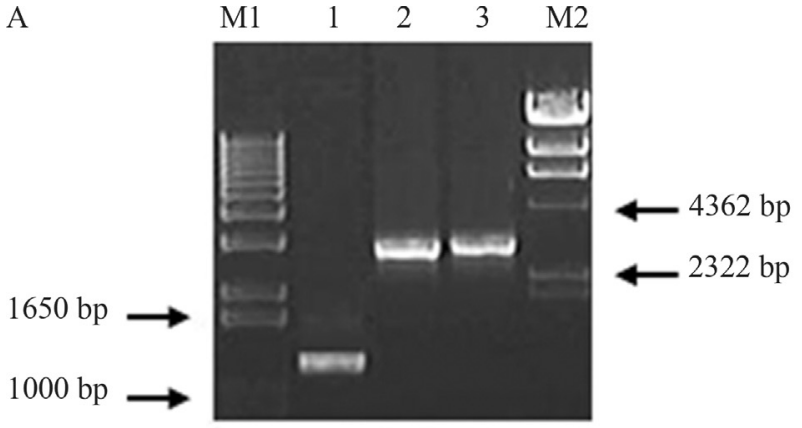

B

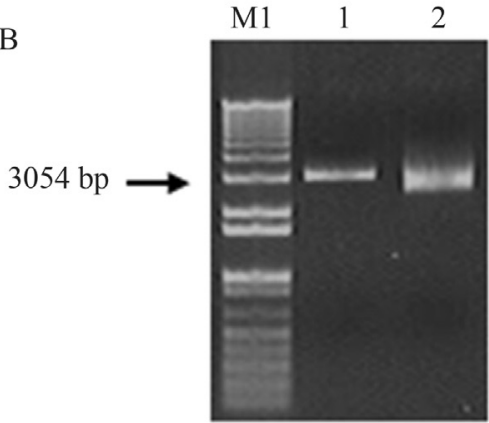

Figure 1 - (A) Amplification of segment A and B using RNA extracted from CEF supernatant inoculated with Wt-IBDV-Br. Segment A was amplified in two overlapping PCR fragments A1 and A2 (1 and 2) with 1173 and 2789 bp, respectively. Segment B was amplified in one PCR product with 2.827 bp (3); (B) Full-length PCR to confirm the segment A (lane 1, $3260 \mathrm{bp}$ ) and B (lane 2, $2827 \mathrm{bp}$ ) cloning in pJG-IBDV-Br-A and pJG-IBDV-Br-B plasmids. M1: 1-kb plus DNA ladder (Invitrogen, Carlsbad, CA, USA); M2: Lambda DNA/HindIII marker (Invitrogen, Carlsbad, CA, USA).

\section{Genetic stability of the recovered virus}

IC-IBDV-Br was propagated in CEF cells (up to 5 passages) and its genome sequence was compared to pJG-IBDV-Br-A and pJG-IBDV-Br-B sequences. The IC-
IBDV-Br sequences were submitted to GenBank (accession number KC603937 and KC603936 to A and B segments, respectively). The analysis of IC-IBDV-Br genome $v s$. pJG-IBDV-Br-A and pJG-IBDV-Br-B plasmids is showed in Table 4. 
Table 3 - Sequence analysis of the pJG-IBDV-Br-A and pJG-IBDV-Br-B plasmids compared to NCBI sequences.

\begin{tabular}{|c|c|c|c|c|c|c|}
\hline \multirow[t]{2}{*}{ Genome region } & \multicolumn{3}{|c|}{ Segment A } & \multicolumn{3}{|c|}{ Segment B } \\
\hline & $\mathrm{nt}^{\mathrm{a}}$ & pJG-IBDV-Br-A & AJ310185.1 ${ }^{\mathrm{f}}$ & $\mathrm{nt}$ & pJG-IBDV-Br-B & EU162095.1 ${ }^{\mathrm{f}}$ \\
\hline \multirow[t]{4}{*}{$\operatorname{UTR}(n t)^{b}$} & 45 & $\mathrm{~g}$ & $\mathrm{c}$ & 41 & a & $\mathrm{t}$ \\
\hline & 49 & $\mathrm{~g}$ & $\mathrm{a}$ & & & \\
\hline & 69 & $\mathrm{a}$ & $\mathrm{g}$ & & & \\
\hline & 102 & $\mathrm{c}$ & $\mathrm{t}$ & & & \\
\hline \multirow[t]{9}{*}{$\operatorname{ORF}(\mathrm{aa})^{\mathrm{c}}$} & $356^{\mathrm{d}}$ & Tyr & Tyr & & & \\
\hline & $649^{\mathrm{d}}$ & Tyr & Tyr & $303^{\mathrm{d}}$ & Pro & Pro \\
\hline & 969 & Thr & Asn & $942^{\mathrm{d}}$ & Ile & Ile \\
\hline & 1107 & Leu & Ser & $999^{\mathrm{d}}$ & Lys & Lys \\
\hline & 1377 & Ile & Thr & $1404^{\mathrm{d}}$ & Ala & Ala \\
\hline & $1765^{\mathrm{d}}$ & Ala & Ala & $1627^{\mathrm{d}}$ & Leu & Leu \\
\hline & $2212^{\mathrm{d}}$ & Ala & Ala & $1720^{\mathrm{e}}$ & Val & Ile \\
\hline & $2235^{\mathrm{e}}$ & Lys & Arg & & & \\
\hline & $2761^{\mathrm{d}}$ & Leu & Leu & & & \\
\hline
\end{tabular}

${ }^{\mathrm{a}}$ Nucleotide.

${ }^{\mathrm{b}}$ Untranslated Region, mutations shows in nucleotides.

'Open reading frame, mutations shown in amino acids.

${ }^{\mathrm{d}}$ Silent mutations.

${ }^{\mathrm{e}}$ Amino acids with the same chemical function.

${ }_{\mathrm{f}}^{\mathrm{A}}$ Access number of the sequences of greatest homology with pJG-IBDV-Br-A and pJG-IBDV-Br-B plasmids (access date 08/15/2012).

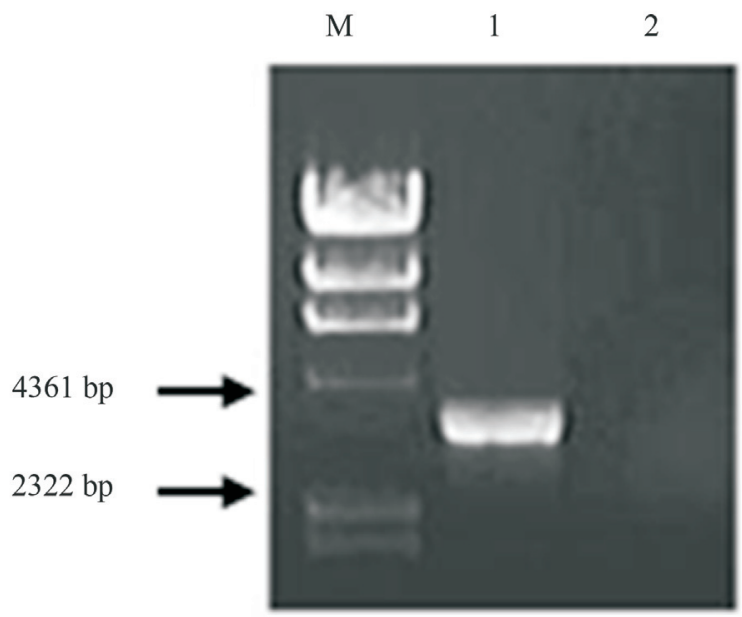

Figure 2 - Amplification of segment A using RNA extracted from clarified supernatant of passage 5 (P5) of the IC-IBDV-Br. The segment A was amplified in one PCR product with $3.260 \mathrm{bp}$ (lane 1). In addition, a PCR was performed without RT reaction to exclude the possibility of amplification might have occurred through residual plasmid DNA (lane 2). M: Lambda DNA/HindIII marker (Invitrogen, Carlsbad, CA, USA).

\section{Discussion}

IBDV is a highly contagious pathogen responsible for economic losses in the poultry industry worldwide. The lesions observed after inoculations of Wt-IBDV-Br in SPF eggs, such as hemorrhage and delayed embryonic development, were similar to the lesions observed by Perera et al. (2002) and similar to first descript for IBDV in Gumboro,

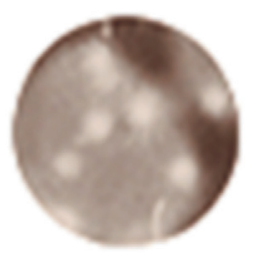

A

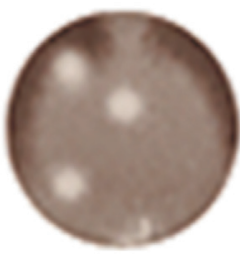

B



C
Figure 3 - Plaque assay for IC-IBDV-Br and Wt-IBDV-Br. CEF cells were inoculated with serial dilutions (10-1 to 10-9) with Wt-IBDV-Br or IC-IBDV-Br. A) Plaque assay for IC-IBDV-Br at 10-6 dilution; B) Plaque assay for Wt-IBDV-Br at 10-7 dilution; C) Negative control inoculated with supernatant of CEF transfected with only pJG-IBDV-Br-A.

USA (Cosgrove, 1962). In addition to suggestive lesions of IBDV infection, the sequence analysis of the VP2 region of isolated virus confirmed the IBDV identity.

The comparison of the segment A in pJG-IBDV-Br-A plasmid vs. AJ310185.1 (NCBI) showed that all observed changes in open reading frame were silent or with aa of the same chemical function, except for the 969, 1107, $1377 \mathrm{nt}$. The 969 and 1377 mutations changes were not observed in any GenBank sequences. However, the 1107 mutation changes already was found in PBG-98 strain, GenBank, access number D00868.1 (access date 10/15/12). The segment B changes in Wt-IBDV-Br clone (pJG-IBDV-Br-B plasmid) $v s$. EU162095.1 (NCBI) were all silent or with aa of the same chemical function. The comparison between the nucleotide sequence of pJG-IBDV-Br-A and pJG- 


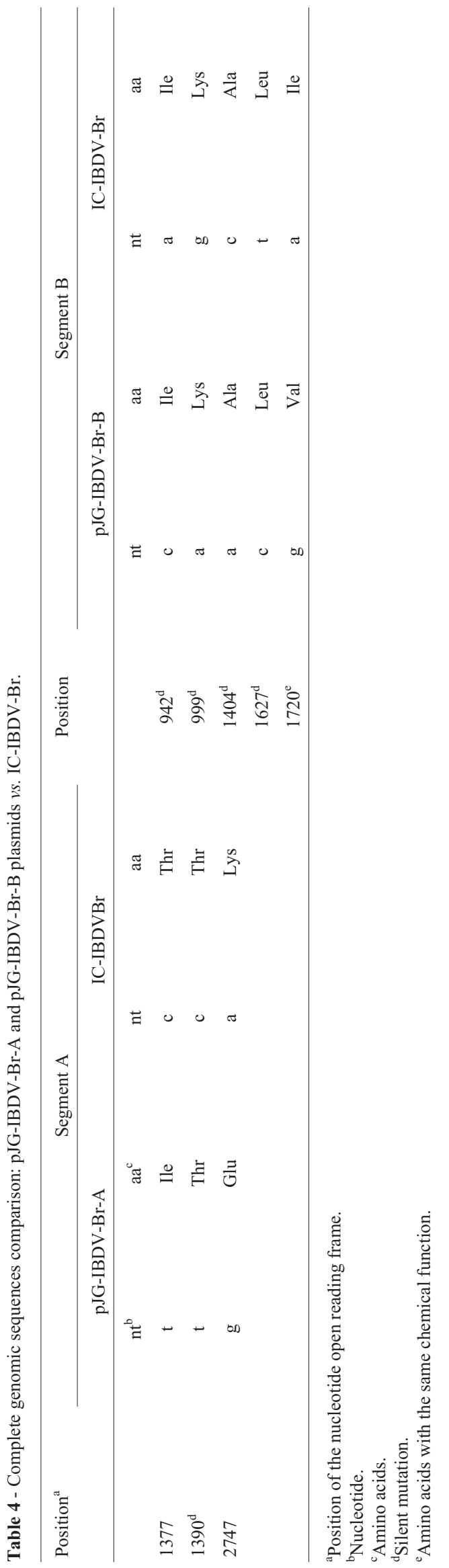

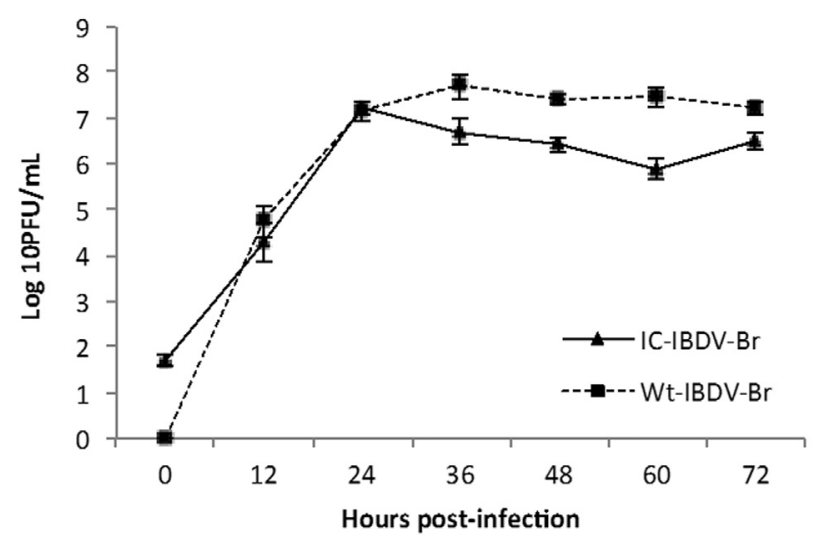

Figure 4 - Comparison of viral growth kinetics between IC-IBDV-Br and Wt-IBDV-Br. CEF cells were inoculated with viruses at MOI of 0.03 $\mathrm{PFU} / \mathrm{mL}$. At different time points indicated, supernatants were collected and infectious virus titers were determined by plaque assay. Each value is represented as mean of the two independent experiments.

IBDV-Br-B plasmids and IC-IBDV-Br virus rescued showed changes of three and five nucleotide in segment $A$ and $\mathrm{B}$, respectively. Interestingly, all nucleotide changes into segment $\mathrm{B}$ in pJG-IBDV-Br-B plasmid $v s$. IC-IBDV-Br rescued virus were silent mutations or for aa of the same chemical function, and all these are also present in EU162095.1 (NCBI).

In order to rule out that the changes were not from the homologous recombination in yeast, the gene regions with these changes were sequenced from Wt-IBDV-Br virus, and it showed the same nucleotides of pJG-IBDV-Br-B plasmid (data not shown). This result supports the stability of virus genome during the cloning process by homologous recombination in yeast.

After the sequencing and genetic characterization of the Wt-IBDV-Br isolated, the viral reverse genetics system was constructed. This system is an important tool for investigations of the viral life cycle and biotechnology application (Gao et al., 2011). The reverse genetics system for IBDV has undergone changes over time. The first reverse genetics system for IBDV was possible by transfection into Vero cells with in vitro transcribed RNA of both segments (Mundt and Vakharia, 1996). An improved technique was followed by transfection of viral cDNA cloned downstream of the T7 (Boot et al., 1999) or CMV promoter (Lim et al., 1999).

The comparison between the two strategies showed the latter as more efficient for generation of IBDV viral particles (Boot et al., 2001). Additional improvements were also followed by addition of ribozyme sequences at both ends (5 and 3) (Qi et al., 2007) or only at the 3 of the cloned DNA (Ben Abdeljelil et al., 2008). Nowadays, the transfection of cloned viral cDNA has been used to evaluate the viral protein influence on IBDV virulence and more recently to development of recombinant viruses for vaccine purposes (Gao et al., 2011). 
However, all assembly of the plasmids used to generate IBDV particles involves multiple rounds of amplification and need of in vitro ligation and restriction sites. Thus, we report the first reverse genetics system for IBDV constructed from homologous recombination in yeast. This method has been described in the literature for the genetic manipulation of other viruses, such as Yellow Fever (Queiroz et al., 2013) and HIV-1 (Marozsan and Arts, 2003) and it has shown to be efficient and easier to perform compared to the use of restriction enzymes and in vitro ligation.

The genetic stable and the similar phenotypes between IC-IBDV-Br and Wt-IBDV-Br confirm the ability of the homologous recombination system in yeast to maintain the characteristics of the parental virus from which the reverse genetics system was built on. The development of this biotechnological resource will help to improve in depth the knowledge on IBDV virology and may be used in vaccines development.

\section{References}

Azad AA, Barrett SA, Fahey KJ (1985) The characterization and molecular cloning of the double-stranded RNA genome of an Australian strain of infectious bursal disease virus. Virology 143:35-44.

Balamurugan V, Kataria JM (2006). Economically important nooncogenic immunosuppressive viral diseases of chickencurrent status. Vet Res Commun 30:541-566.

Ben Abdeljelil N, Khabouchi N, Mardassi H (2008) Efficient rescue of infectious bursal disease virus using a simplified RNA polymerase II-based reverse genetics strategy. Arch Virol 153:1131-1137.

Boot HJ, Dokic K, Peeters BP (2001) Comparison of RNA and cDNA transfection methods for rescue of infectious bursal disease virus. J Virol Methods 97:67-76.

Boot HJ, ter Huurne AA, Peeters BP, Gielkens, AL (1999) Efficient Rescue of Infectious Bursal Disease Virus from Cloned cDNA: Evidence for Involvement of the 3'-Terminal Sequence in Genome Replication. Virology 265:330-341.

Cosgrove AS (1962) An apparently new disease of chickens avian nephrosis. Avian Dis 6:385-389.

Di Fabio J, Rossini LI, Eterradossi N, Toquin MD, Gardin Y (1999) European - like infectious bursal disease viruses in Brasil. Vet Rec 145:203-204.

Gao L, Qi X, Li K, Gao H, Gao Y, Qin L, Wang Y, Wang X (2011) Development of a tailored vaccine against challenge with very virulent infectious bursal disease virus of chickens using reverse genetics. Vaccine 29:5550-5557.

Gibson DG (2009) Synthesis of DNA fragments in yeast by one-step assembly of overlapping oligonucleotides. Nucleic Acids Res 37:6984-6990.

Hudson PJ, McKern NM, Power BE, Azad AA (1986) Genomic structure of the large RNA segment of infectious bursal disease virus. Nucleic Acids Res 14:5001-5012.

Jackwood, D. J., Y. M. Saif, and J. H. Hughes. 1982. Characteristics and serologic studies of two serotypes of infectious bursal disease virus in turkeys. Avian Dis 26:871-882.
Lim BL, Cao Y, Yu T, Mo CW (1999) Adaptation of very virulent infectious bursal disease virus to chicken embryonic fibroblasts by site-directed mutagenesis of residues 279 and 284 of viral coat protein VP2. J Virol 73:2854-2862.

Marozsan AJ, Arts EJ (2003) Development of a yeast-based recombination cloning/system for the analysis of gene products from diverse human immunodeficiency virus type isolates. J Virol Methods 111:111-120.

Mundt E, Beyer J, Müller H (1995) Identification of a novel viral protein in infectious bursal disease virus-infected cells. J Gen Virol 76:437-443.

Mundt E, Vakharia VN (1996) Synthetic transcripts of doublestranded Birnavirus genome are infectious. Proc Natl Acad Sci U S A 93:11131-11136.

Müller H, Scholtissek C, Becht H (1979) The genome of infectious bursal disease virus consists of the two segments of double-stranded RNA. J Virol 31:584-589.

Nakano MP, MASC, Saliba AM, Nobre D, Narimatsu MN (1972) Ocorrência da "Doença de Gumboro" no Brasil. Diagnóstico anátomo-patológico. Biológico 38:60-61.

Oldenburg KR, Vo KT, Michaelis S, Paddon C (1997) Recombination-mediated PCR-directed plasmid construction in vivo in yeast. Nucleic Acids Res 25:451-452.

Pan J, Vakharia VN, Tao YJ (2007) The structure of a birnavirus polymerase reveals a distinct active site topology. Proc Natl Acad Sci U S A 104:7385-7390.

Perera CL, Noda J, Coroas LI, Alfonso P, Cuello S (2002) Caracterización de la cepa BF8 del virus de la enfermedad infecciosa de la bolsa. Revista Cubana de Ciencia Avícola 26:111-119.

Qi X, Gao Y, Gao H, Deng X, Bu Z, Wang X, Fu C, Wang X (2007) An improved method for infectious bursal disease virus rescue using RNA polymerase II system. J Virol Methods 142:81-88.

Queiroz SRA, da Silva ANMR, Santos JJS, Marques Junior E, Bertani GR, Gil LHVG (2013) Construction of yellow fever virus subgenomic replicons by yeast-based homologous recombination cloning technique. Anais da Academia Brasileira de Ciências 85:159-168.

Reed LJ, Muench H (1938) A simple method of estimating fifty percent endpoints. Am J Hyg 27:493-497.

Reynolds DL (1998) Enteric Viruses. A Laboratory Manual for the Isolation and Identification of Avian Pathogens. 4th edition. American Association of avian pathologists, Florida.

Romero CH (1987) Manual de técnicas virológicas e sorológicas para o isolamento e identificação de vírus aviários e seus anticorpos. Embrapa - Centro Nacional de Pesquisa de Suínos e Aves, Concórdia.

Sambrook J, Russell, DW (2001) Molecular Cloning: A Laboratory Manual. 3rd edition. Cold Spring Harbor Laboratory Press, New York.

Saukas TN (1978) Caracterização de amostra do vírus da doença infecciosa bursal (doença de Gumboro) isolada no Brasil, Rio de Janeiro, Brasil. M.Sc. Dissertation, Universidade Rural do Rio de Janeiro, 88p.

Shanks RM, Kadouri DE, MacEachran DP, O'Toole GA (2009) New yeast recombineering tools for bacteria. Plasmid 62:88-97.

Wang YS, Ouyang W, Liu XJ, He KW, Yu SQ, Zhang HB, Fan HJ, Lu CP (2012) Virus-like particles of hepatitis B virus core protein containing five mimotopes of infectious bursal 
disease virus (IBDV) protect chickens against IBDV. Vaccine 30:2125-2130.

von Einem UI, Gorbalenya AE, Schirrmeier H, Behrens SE, Letzel T, Mundt E (2004) VP1 of infectious bursal disease virus is a RNA-dependent RNA polymerase. J Gen Virol 85:2221-2229.
Zanetti FA, Del Médico Zajac MP, Taboga OA, Calamante G (2012) Evaluation of modified vaccinia virus Ankara expressing VP2 protein of infectious bursal disease virus as an immunogen in chickens. J Vet Sci 13:199-201.

All the content of the journal, except where otherwise noted, is licensed under a Creative Commons License CC BY-NC. 